\title{
A comparison of catch phase force-time characteristics during clean derivatives from the knee
}

Submission type: Original investigation

Running title: $\quad$ Clean catch phase force-time characteristics

Authors: $\quad$ Paul Comfort ${ }^{1}{ }^{*}$, Robert Williams ${ }^{1}$, Timothy J. Suchomel ${ }^{2} \&$ Jason P. Lake $^{3}$

\section{Researchers affiliations:}

${ }^{1}$ Directorate of Sport, Exercise and Physiotherapy, Human Performance Laboratory, University of Salford, Salford, UK

${ }^{2}$ Department of Human Movement Sciences, Carroll University, Waukesha, WI, USA

${ }^{3}$ Department of Sport and Exercise Sciences, University of Chichester, Chichester, West Sussex, UK

Corresponding Author* Paul Comfort, University of Salford, Frederick Road, Salford, Greater

Manchester, UK. Telephone: 0044161295 6358. Fax: 00441612952673

E-mail: p.comfort@salford.ac.uk 


\section{Abstract}

2 The aim of this study was to compare load-absorption force-time characteristics of the clean

3 from the knee $(\mathrm{CK})$, power clean from the knee (PCK) and clean pull from the knee (CPK).

4 Ten collegiate athletes (age $27.5 \pm 4.2$ years; height $180.4 \pm 6.7 \mathrm{~cm}$; mass $84.4 \pm 7.8 \mathrm{~kg}$ ),

5 performed three repetitions each of the CK, PCK and CPK with 90\% of their 1RM power

6 clean on a force platform. The CK load-absorption duration $(0.95 \pm 0.35 \mathrm{~s})$ was significantly

7 longer compared to the CPK $(0.44 \pm 0.15 \mathrm{~s} ; p<0.001, d=2.53)$, but not compared to the

8 PCK $(0.56 \pm 0.11 \mathrm{~s} ; p>0.05, d=1.08)$, with no differences between PCK and CPK $(p>$

$90.05, d=0.91)$. The CPK demonstrated the greatest mean force $(2039 \pm 394 \mathrm{~N})$, which was significantly greater than the PCK $(1771 \pm 325 \mathrm{~N} ; p=0.012, d=0.83)$, but not significantly different to the CK $(1830 \pm 331 \mathrm{~N} ; p>0.05, d=0.60)$; CK and PCK were not different $(p>$ $0.05, d=0.18)$. Significantly more load-absorption work was performed during the CK (655 $\pm 276 \mathrm{~J})$ compared to the PCK $(288 \pm 109 \mathrm{~J} ; d=1.75, p<0.001)$; but not compared to the

CPK $(518 \pm 132 \mathrm{~J} ; d=0.80, p>0.05)$. Additionally, more load-absorption work was performed during the CPK compared to the PCK $(d=1.90, p=0.032)$. Inclusion of the catch phase during the $\mathrm{CK}$ does not provide any additional stimulus in terms of mean force or work during the load-absorption phase compared to the CPK, while the CPK may be beneficial in training rapid force absorption due to high force and a short duration.

Key words: weightlifting derivatives; power clean from the knee; clean pull from the knee; eccentric loading 
Lower body force and power development are essential for improving athlete performance during tasks that require rapid extension of the hip, knee, and ankle joints $(10,28)$. Various training methods, including plyometric exercises (1, 2, 26), kettlebell training $(19,22)$, strength training $(4,9)$ and the use of weightlifting exercises and their derivatives $(4,17,22$, 36) have been reported to enhance these qualities. Of these training methods, investigators have reported that the inclusion of weightlifting derivatives results in superior performance improvements compared to other training methods $(17,22,36)$. It is therefore not surprising that weightlifting derivatives are commonly incorporated into athletes' training programs.

Research into the biomechanics of weightlifting derivatives has shown that the second pull phase of the clean and snatch results in the greatest net vertical force and power applied to the barbell $(12,13,16)$. When comparing the power clean, power clean from the knee (PCK), mid-thigh power clean, and mid-thigh pull, researchers have observed that the greatest force and power applied to the system occurs during the mid-thigh power clean and the mid-thigh pull, with no differences between the two mid-thigh variations $(5,6)$. In addition, Suchomel and colleagues (35) reported greater force, impulse, rate of force development and power during the jump shrug compared to the hang power clean and hang high pull. Such findings indicate that the pulling phase of weightlifting movements may be the most beneficial component of such exercises when focusing on maximal force and power development. This is supported by a recent review which concluded that eliminating the catch phase may decrease lift complexity, resulting in greater coaching efficiency in athletes with limited experience of the full lifts, possibly reducing injury risk (29) as most of the reported injuries occur to the hand, arm, and trunk $(21,24,27)$. In addition, excluding the catch phase permits the use of higher loads (i.e. greater than one repetition maximum power clean), which has 
It has been suggested that the catch phase of the clean and power clean may be important in developing an athletes' capacity to cope with the mechanical demands of impact (20). However, only one study has investigated the work performed during the catch phase, demonstrating that the total work during the clean was greater than the power clean, although this was similar to the total work during a drop landing (20). It is worth noting however, that these results may vary in stronger lifters as the relative one repetition maximum (1RM) clean in the study above was only $0.86 \pm 0.12 \mathrm{~kg} / \mathrm{kg}$ of body mass. The similarity in the work performed between the drop landing and the clean may be explained by the fact that the barbell is caught just below its peak vertical displacement during the clean (15) and therefore does not add substantially to the mass that has to be decelerated.

While researchers have compared the force-time characteristics of the concentric phase of weightlifting derivatives as previously mentioned, no research to date has examined differences between the force-time characteristics of the catch phase of weightlifting derivatives. It is important to note that because some weightlifting derivatives do not include a traditional catch phase (e.g. weightlifting pulling derivatives), terms such as the 'loadabsorption' phase may describe this part of the lift more effectively. There is currently a need to establish whether the force-time characteristics of weightlifting derivative load absorption phases are comparable so that practitioners can make informed decisions about what exercise(s) should be prescribed to develop the athlete's ability to cope with the mechanical demands of the load absorption phase. This information could also enable practitioners to make informed decisions about which weightlifting derivatives to prescribe during different phases of the athlete's periodized training plan. The aim of this study therefore, was to compare force-time characteristics of the load-absorption phase of the clean from the knee (CK), PCK, and clean pull from the knee (CPK) to determine and compare their mechanical demands. It was hypothesized that the greatest demands would occur during the CK due to 
the increased displacement of the system center of mass (body plus barbell) compared to the PCK and CPK equivalent, in line with previous observations (20).

\section{Methods}

\section{Experimental Approach to the Problem}

A within subject repeated measures design was used to test our hypotheses. Subjects performed CK, PCK, and CPK, with $90 \%$ of their 1RM power clean, in a randomized order while standing on a force platform that recorded force-time data. Duration, mean force, and work, during the load-absorption phase, were calculated from the force-time data and compared to establish the effect of exercise. The duration of the load-absorption phase was examined to determine the length of time over which force was produced in order to decelerate the system center of mass during each weightlifting derivative. Load-absorption mean force was examined to provide a greater understanding of the magnitude of force the athlete is exposed to over the entire duration of this phase during each weightlifting derivative. Finally, work performed during the load-absorption phase of each weightlifting derivative was studied to establish the effect that exercise had on the absorption of potential energy following the second pull.

\section{Subjects}

Ten male collegiate level team sport (rugby league, rugby union, soccer) athletes (age $27.5 \pm$ 4.2 years; height $180.4 \pm 6.7 \mathrm{~cm}$; mass $84.4 \pm 7.8 \mathrm{~kg}$; relative $1 \mathrm{RM}$ power clean $1.28 \pm 0.18$ $\mathrm{kg} / \mathrm{kg}$ of body mass), who regularly performed weightlifting derivatives ( $\geq 3$ times per week, for $\geq 2$ years), volunteered to participate. They were free from injury and provided written 
97 informed consent. This investigation received ethical approval from the institutional review board and conformed to the World Medical Association declaration of Helsinki. Subjects were requested to perform no strenuous exercise during the 48 hours prior to testing, maintain their normal dietary intake prior to each session, and to attend testing sessions in a hydrated state.

\section{Procedures}

Before experimental trials, subjects visited the laboratory on two occasions, at the same time of day (5-7 days apart), to establish the reliability of power clean 1RM, following the protocol of Baechle, Earle and Wathen (3). All power clean attempts began with the barbell on the lifting platform, and ended with the barbell caught on the anterior deltoids in a semisquat position; $>90^{\circ}$ internal knee angle (any attempt caught below this angle was disallowed). All testing was performed using a lifting platform (Power Lift, Jefferson, USA), weightlifting bar and plates (Werksan, New Jersey, USA). The greatest load achieved across the two sessions was used to calculate the load used during the CK, PCK and CPK.

\section{Subjects returned to the laboratory 5-7 days after the second 1RM testing session, and} performed a standardized warm up including body weight squats, lunges and dynamic stretching. This was followed by performance of the CK, PCK, and CPK with progressively heavier loads $(45,60,75 \% 1 \mathrm{RM}$ power clean) prior to performing three single lifts of each of the CK variations (a total of nine repetitions), in a randomized order, with $90 \%$ of $1 \mathrm{RM}$ power clean. This load was used as this represents the upper range of the loads usually recommended for the clean and power clean from the knee and such loads are more likely to ensure that the subjects received the bar at the bottom of the clean, whereas at lower loads it is more likely 
that the subjects may catch the bar prior to completing the descent into the clean catch position, which would have resulted in additional repetitions to be performed and increase the chance of fatigue influencing the results. Two minutes of rest was provided between repetitions, and five minutes between lifts. The CK, PCK, and CPK were performed using previously described technique $(11,33)$. Each variation started from a static position with the barbell located at the top of the patella. Subjects then transitioned to the mid-thigh position before performing triple extension at the hip, knee, and ankle joints (i.e. second pull) in one continuous rapid movement. During the CK and PCK, the barbell was elevated and caught in the rack position in a full depth squat (thighs below parallel to the floor) or in the rack position in a shallow squat ( $>90^{\circ}$ internal degree knee angle), respectively. In contrast, the CPK required subjects to perform the transition and second pull and then control and decelerate the barbell as it descended from its maximum height. All CK variations were performed while subjects stood on a force platform (Kistler, Winterthur, Switzerland, Model 9286AA, SN 1207740) recording vertical force at $1000 \mathrm{~Hz}$ with Bioware software (Version 5.0.3: Kistler Instruments Corporation).

\section{Data Analysis}

Unfiltered force-time data were exported from Bioware and analyzed using custom LabVIEW software (Version 10.0; National Instruments, Austin, TX, USA). Force-time data from all trials were analyzed to obtain the dependent variables and were averaged for statistical analysis. The dependent variables were: loading duration, mean force, and work. Transition from pulling to load-absorption was represented by two distinct force-time curves (Figures 1-3); the most obvious where subjects left the ground (Figures $1 \& 2$ ), and when this occurred a force threshold of $10 \mathrm{~N}$ was used to indicate both take off and load-absorption. 
This was used because pilot testing showed that the method recently described and used by Owen et al. (23) to identify the start of the CMJ (1 s mean force \pm 5 SD) typically fell between 5 and $10 \mathrm{~N}$ when applied to the mid-part of flight time (flight time less the first and last $0.03 \mathrm{~s}$ ). When subjects did not leave the ground, the lowest post-pull force was identified and the same $10 \mathrm{~N}$ threshold used to identify the beginning of load-absorption (Figure 3). Load-absorption ended when system center of mass displacement reached zero (See Figures 1 \& 2). Mean force during load-absorption was calculated by averaging force over this phase. Load absorption system center of mass displacement was calculated by subtracting the position of the system center of mass at the end of this phase from its position at the beginning of this phase. Load-absorption work was calculated by multiplying load-absorption mean force by load-absorption displacement.

*** Insert Figure 1, $2 \& 3$ about here***

\section{Statistical Analyses}

Inter-repetition consistency for load-absorption duration, mean force, and work for each CK variation were determined using intraclass correlation coefficients (ICC). Distribution of data was analyzed via Shapiro-Wilks' test of normality. Exercise effect on the dependent variables was analyzed using a one-way repeated measures analysis of variance (ANOVA) including Bonferroni post-hoc analysis. An a priori alpha level was set at $p \leq 0.05$. The magnitude of differences was determined via calculation of Cohen's $d$ effect sizes, which were interpreted 
based on the recommendations of Rhea et al. (25), where $<0.35,0.35-0.80,0.80-1.50,>1.50$ are considered trivial, small, moderate and large, respectively.

\section{Results}

Power clean $1 \mathrm{RM}$ performances were highly reliable $(\mathrm{ICC}=0.997)$ between sessions one $(107.2 \pm 14.3 \mathrm{~kg})$ and two $(108.0 \pm 15.1 \mathrm{~kg})$. All dependent variables demonstrated moderate to high reliability between trials, across each of the three CK variations (Table 1).

***Insert Table 1 about here***

Load-absorption duration was significantly different $(p<0.001$, Power $=0.995)$ across CK variations; post hoc analysis showed that $\mathrm{CK}$ load-absorption duration $(0.95 \pm 0.35 \mathrm{~s})$ was significantly longer than CPK load-absorption duration $(0.44 \pm 0.15 \mathrm{~s} ; p<0.001, d=2.53)$, and moderately although not significantly longer than PCK load-absorption duration $(0.56 \pm$ $0.11 \mathrm{~s} ; p>0.05, d=1.08$ ) (Figure 3 ). There were no differences between PCK and CPK loadabsorption duration $(p>0.05, d=0.91)$ (Figure 4).

*** Insert figure 4 about here $* * *$ 
Mean force during the load-absorption phase was significantly different $(p=0.015$, Power $=$ 0.678) across CK variations; CPK demonstrated the highest mean force $(2039 \pm 394 \mathrm{~N})$, which was moderately and significantly greater than the PCK mean force $(1771 \pm 325 \mathrm{~N} ; p=$ $0.012, d=0.83)$, but not significantly different compared to the CK mean force $(1830 \pm 3301$ $\mathrm{N} ; p>0.05, d=0.60)$ (Figure 5). There were no differences between CK and PCK values ( $p$ $>0.05, d=0.18)$ (Figure 5).

$* * *$ Insert figure 5 about here***

201

202

203 Work during the load-absorption phase was significantly $(p=0.001$, Power $=0.993)$ different across CK variations. Significantly more work occurred during the load-absorption phase of the CK $(655 \pm 276 \mathrm{~J})$ compared to the PCK $(288 \pm 109 \mathrm{~J} ; p<0.001, d=1.75)$, but was not significantly different from the CPK $(518 \pm 132 \mathrm{~J} ; p>0.05, d=0.80)$ (Figure 6). Significantly more work was performed during the CPK compared to the PCK $(p=0.032, d$ $=1.90)$ (Figure 6). 


\section{Discussion}

213 The purpose of this study was to compare the force-time characteristics of the loadabsorption phase of the CK, PCK, and CPK. The three primary findings of the current study are as follows: first, CK load-absorption duration was significantly longer compared to the CPK, as hypothesized, but was not significantly different compared to the PCK; second, CPK load-absorption mean force was significantly larger compared to the PCK, but was not significantly different compared to the CK; finally, more work was performed during CK load-absorption compared to the PCK, while there was no significant difference regarding the work performed during CK and CPK load-absorption.

In line with our hypothesis, the CK produced the longest load-absorption duration of all of the examined CK variations. Although not significantly different from the PCK loadabsorption duration, the effect size was moderate, indicating that this is a practically meaningful effect. In contrast, a large practically meaningful difference was present between CK and CPK load-absorption duration. These findings should come as no surprise given the demands of each exercise. Compared to the PCK and CPK that finish with the athlete in semi-squat position $(11,33)$, the $\mathrm{CK}$ requires an athlete to drop under the bar and rack it across their shoulders while descending into a full depth front squat position. Due to its duration, CK load-absorption may permit an athlete to absorb the forces more efficiently compared to the PCK and CPK, which may require a more rapid absorption of the external load over a smaller displacement. This is supported by previous research that suggested that the clean enables greater energy absorption when compared to the power clean (20). 
The results of the current study indicated that the CPK resulted in the greatest mean forces during the load-absorption phase, which is in contrast to our hypothesis. Only one previous study had measured the force production characteristics of a weightlifting pulling derivative following the second pull or propulsion phase (34). However, that study focused on peak landing forces of a single exercise instead of comparing the differences between several exercises. When compared to CK and PCK load-absorption mean force, the CPK demonstrated small and moderately higher mean force, respectively. This is a unique finding in the sense that the load deceleration position of the CPK (i.e. mid-thigh position) may enable the athlete to experience greater force acceptance in a position that is considered to be the strongest and most powerful position during the concentric phase of the weightlifting derivatives (12-14). A reported benefit of the catch phase of weightlifting derivatives is the rapid acceptance of an external load (29). There have been arguments that the catch phase may simulate impact absorption in sports such as American football; however, there is no research to support the efficacy of this claim. In fact, the results of the current study show that the CPK may simulate the rapid acceptance of a load to a greater extent than the CK and PCK. These findings may have training implications as the CPK may facilitate the use of loads in excess of power clean 1RM (11). Such loading has been shown to emphasize force production during the propulsion phase of weightlifting movements $(7,8,18)$, but may also provide comparable or greater mean force production during the load-absorption phase following the second pull. Ultimately, this may enable the athlete to further develop the magnitude and rate of force production during the concentric and eccentric phases of the lift.

Previous research indicated that the work completed during the load-absorption phase of weightlifting derivatives may improve the capacity to absorb forces during impact tasks (20). Similar to the study of Moolyk et al. (20), the current study indicated that the CK resulted in significantly more work compared to the PCK. This is likely due to the longer load- 
absorption duration, greater load-absorption mean force, and because of the requirements of the $\mathrm{CK}$ a greater lifter center of mass displacement during the catch (although this was not assessed during this study). It is worth noting that the barbell is generally caught just below its peak vertical displacement during the clean (15), and therefore does not add substantially to the mass that has to be decelerated; however, the displacement of the lifter's centre of mass is much greater after the second pull during the $\mathrm{CK}$ compared to the $\mathrm{PCK}$ and $\mathrm{CPK}$. From a practical standpoint, a weightlifting derivative performed through a full range of motion may be used to develop the strength and flexibility needed to absorb the forces experienced during landing tasks (20). However, a unique finding of the current study was the fact that the work performed during the load-absorption phase of the CPK was not significantly different from the CK, although, a small to moderate effect was present. The similarities in work may be explained by the differences in mean force and duration; however, further research is warranted to deconstruct these findings and their potential application in training.

The use of weightlifting pulling derivatives in strength and conditioning programs has been discussed in a recent review (29), although intervention studies are required to confirm the potential benefits of such training. While previous research on weightlifting pulling derivatives has focused on the second pull or propulsion phase of the movements $(5-8,30-32$, 35), less is known about the load-absorption phase of these lifts. A recent study by Suchomel et al. (34) examined the landing forces of the jump shrug across several different loads. Their results indicated that landing force decreases as external load increases, indicating that the forces experienced during the landing should not deter a practitioner from prescribing heavier loads. Although this information is beneficial from an exercise prescription standpoint, the current study is the first of its kind to examine more descriptive variables that characterize the load-absorption phase of weightlifting derivatives. Collectively, the results of the current study indicate that the CPK may produce similar mean forces and work during the load- 
absorption phase, while also including a shorter load-absorption duration, compared to the

284 CK. Practically speaking, it appears that the CPK may benefit not only the force and power production during extension of the hips, knees and ankles, but also the necessary forces needed to subsequently decelerate the load of the lifter and barbell.

287

The findings of the current study are not without their limitations. The reliability of the CK load-absorption duration was poor compared to the other CK variations. It is possible that despite the subjects' experience with CK variability in the full front squat catch position may have occurred. This idea is supported by the standard deviations for loading duration observed in this study. A second limitation may be the exclusion of joint kinetic and kinematic measurements. While this limitation does not lessen the value of lifter plus barbell system measurements, future research should consider examining similar research questions using 3D motion analysis to determine whether similar trends exist at the joint level. Furthermore, future research should consider the effect of load on the force-time characteristics of the load-absorption phase of weightlifting derivatives. The information within the current study combined with joint-level measurements may provide a better understanding of the similarities and differences between the load-absorption phase of weightlifting derivatives.

\section{Practical Application}

Although it can be argued that the catch phase trains the ability to transition from rapid extension of hips, knees and ankles against an external load, to rapid flexion of hips, knees and ankles, there appears to be no additional mechanical benefit to including the catch phase, in terms of load-absorption mean force or work, when comparing the CK and CPK performed at $90 \%$ of $1 \mathrm{RM}$ power clean. However, although not presented in this study, it is reasonable 
to assume that total work during the $\mathrm{CK}$ would be greater than compared to the CPK as the

308

309

310

311

312

313

314

315

316

317

318

319

320

321

322

323

324

325

326

\section{9}

20

22 athlete has to stand from a full depth front squat position during the CK. It is suggested the CPK be used during maximum strength mesocycle due to the potential to use loads $>1 \mathrm{RM}$ power clean and during competition phases of training due to the lower volume of work required across the entire lift and the corresponding reduction in injury potential due to the elimination of the catch phase.

The results of the current study do not constitute endorsement of the product by the authors, the journal, or the NSCA.

7

No funding was received to support this study. The authors have no conflict of interest. 


\section{References}

328 1. Adams K, O'Shea J, O'Shea K, and Climstein M. The effect of six weeks of squat, plyometric

and squat plyometric training on power production. J Appl Sports Sci Res 6: 36-40, 1992.

2. Arabatzi F, Kellis E, and Saez De Villarreal E. Vertical Jump Biomechanics after Plyometric, Weight Lifting, and Combined (Weight Lifting + Plyometric) Training. J Strength Cond Res 24: 2440-2448 2010.

3. Baechle TR, Earle RW, and Wathen D. Resistance Training, in: Essentials of Strength Training and Conditioning. TR Baechle, Earle, R. W, ed. Champaign, Illinois: Human Kinetics, 2008, pp 381-412.

4. Channell BT and Barfield JP. Effect of Olympic and Traditional Resistance Training on Vertical Jump Improvement in High School Boys. J Strength Cond Res 22: 1522-1527, 2008.

5. Comfort P, Allen M, and Graham-Smith P. Comparisons of peak ground reaction force and rate of force development during variations of the power clean. $J$ Strength Cond Res 25: 1235-1239, 2011.

6. Comfort P, Graham-Smith P, and Allen M. Kinetic comparisons during variations of the Power Clean. J Strength Cond Res 25: 3269-3273, 2011.

7. Comfort P, Jones PA, and Udall R. The effect of load and sex on kinematic and kinetic variables during the mid-thigh clean pull. Sports Biomech 14: 139-156, 2015.

8. Comfort $\mathrm{P}$, Udall $\mathrm{R}$, and Jones $\mathrm{P}$. The affect of loading on kinematic and kinetic variables during the mid-thigh clean pull. J Strength Cond Res 26: 1208-1214, 2012.

9. Cormie P, McGuigan MR, and Newton RU. Adaptations in athletic performance after ballistic power versus strength training. Med Sci Sports Exerc 42: 1582-1598, 2010.

10. Cormie P, McGuigan MR, and Newton RU. Developing Maximal Neuromuscular Power: Part 2 - Training Considerations for Improving Maximal Power Production. Sports Med 41: 1251462011.

11. DeWeese BH, Suchomel TJ, Serrano AJ, Burton JD, Scruggs SK, and Taber CB. The pull from the knee: Proper technique and application. Strength \& Conditioning Journal 38: 79-85, 2016.

12. Enoka RM. The pull in olympic weightlifting. Med Sci Sports 11: 131-137, 1979.

13. Garhammer J. Power production by Olympic weightlifters. Med Sci Sports Exerc 12: 54-60, 1980.

14. Garhammer J. Energy flow during Olympic weight lifting. Med Sci Sports Exerc 14: 353-360, 1982.

15. Garhammer J. Biomechanical profiles of Olympic weightlifters. Int J Sports Biomech 1: 122130, 1985.

16. Garhammer J. A comparison of maximal power outputs between elite male and female weightlifters in competition. Int J Sports Biomech 3: 3-11, 1991.

17. Hoffman JR, Cooper J, Wendell M, and Kang J. Comparison of Olympic vs. traditional power lifting training programs in football players. J Strength Cond Res 18: 129-135, 2004.

18. Kawamori N, Rossi SJ, Justice BD, Haff EE, Pistilli EE, O'Bryant HS, Stone MH, and Haff GG. Peak Force and Rate of Force Development During Isometric and Dynamic Mid-Thigh Clean Pulls Performed At Various Intensities. J Strength Cond Res 20: 483-491, 2006.

19. Lake JP and Lauder MA. Kettlebell swing training improves maximal and explosive strength. J Strength Cond Res 26: 2228-2233, 2012.

20. Moolyk AN, Carey JP, and Chiu LZF. Characteristics of Lower Extremity Work During the Impact Phase of Jumping and Weightlifting. J Strength Cond Res 27: 3225-3232, 2013.

21. Myer GD, Quatman CE, Khoury J, Wall EJ, and Hewett TE. Youth Versus Adult Weightlifting Injuries Presenting to United States Emergency Rooms: Accidental Versus Nonaccidental Injury Mechanisms. J Strength Cond Res 23: 2054-2060 2009. 
22. Otto WH, III, Coburn JW, Brown LE, and Spiering BA. Effects of Weightlifting vs. Kettlebell Training on Vertical Jump, Strength, and Body Composition. J Strength Cond Res 26: 11991202, 2012.

23. Owen NJ, Watkins J, Kilduff LP, Bevan HR, and Bennett MA. Development of a criterion method to determine peak mechanical power output in a countermovement jump. $J$ Strength Cond Res 28: 1552-1558, 2014.

24. Quatman CE, Myer GD, Khoury J, Wall EJ, and Hewett TE. Sex Differences in Weightlifting: Injuries Presenting to United States Emergency Rooms. J Strength Cond Res 23: 2061-2067 2009.

25. Rhea MR. Determining the Magnitude of Treatment Effects in Strength Training Research Through the Use of the Effect Size. J Strength Cond Res 18: 918-920, 2004.

26. Saez de Villarreal E, Requena B, Izquierdo M, and Gonzalez-Badillo JJ. Enhancing sprint and strength performance: Combined versus maximal power, traditional heavy-resistance and plyometric training. J Sci Med Sport 16: 146-150, 2012.

27. Stone MH, Fry AC, Ritchie M, Stoessel-Ross L, and Marsit JL. Injury Potential and Safety Aspects of Weightlifting Movements. Strength \& Conditioning Journal 16: 15-21, 1994.

28. Stone MH, O'Bryant HS, McCoy L, Coglianese R, Lehmkuhl M, and Schilling B. Power and Maximum Strength Relationships During Performance of Dynamic and Static Weighted Jumps. J Strength Cond Res 17: 140-147, 2003.

29. Suchomel T, Comfort $P$, and Stone M. Weightlifting Pulling Derivatives: Rationale for Implementation and Application. Sports Med 45: 823-839, 2015.

30. Suchomel TJ, Beckham GK, and Wright GA. Lower body kinetics during the jump shrug: impact of load. Journal of Trainology 2: 19-22, 2013.

31. Suchomel TJ, Beckham GK, and Wright GA. The impact of load on lower body performance variables during the hang power clean. Sports Biomech 13: 87-95, 2014.

32. Suchomel TJ, Beckham GK, and Wright GA. The effect of various loads on the force-time characteristics of the hang high pull. J Strength Cond Res 29: 1295-1301, 2015.

33. Suchomel TJ, DeWeese BH, and Serrano AJ. The power clean and power snatch from the knee. Strength \& Conditioning Journal: In Press, 2016.

34. Suchomel TJ, Taber CB, and Wright GA. Jump Shrug Height and Landing Forces Across Various Loads. Int J Sports Physiol Perform 11: 61-65, 2016.

35. Suchomel TJ, Wright GA, Kernozek TW, and Kline DE. Kinetic Comparison of the Power Development Between Power Clean Variations. J Strength Cond Res 28: 350-360, 2014.

36. Tricoli V, Lamas L, Carnevale R, and Ugrinowitsch C. Short-Term Effects on Lower-Body Functional Power Development: Weightlifting Vs.Vertical Jump Training Programs. J Strength Cond Res 19: 433-437, 2005. 
Table 1: Reliability (ICC) of load-absorption phase variables across clean variations from the 431 knee

433 Figure 1: Example CK force-time displacement-time curve

435 Figure 2: Example PCK force-time and displacement-time curve 436

437 Figure 3: Example CPK force-time and displacement-time curve 438

Figure 4: Comparison of load-absorption duration across clean variations from the knee

440

441 Figure 5: Comparison of mean force during the load-absorption across clean variations from 442 the knee

443

444 Figure 6: Comparison of work during the load-absorption across clean variations from the 445 knee 
Table 1: Reliability (ICC) of load-absorption phase variables across lifts

\begin{tabular}{lccc}
\hline Variable & CK & PCK & CPK \\
\hline Loading Duration & 0.645 & 0.713 & 0.958 \\
Loading Mean Force & 0.996 & 0.987 & 0.963 \\
Loading Work & 0.926 & 0.915 & 0.929 \\
\hline
\end{tabular}

Notes: $\mathrm{CK}=$ clean from the knee; PCK = power clean from the knee; CPK = clean pull from the knee 


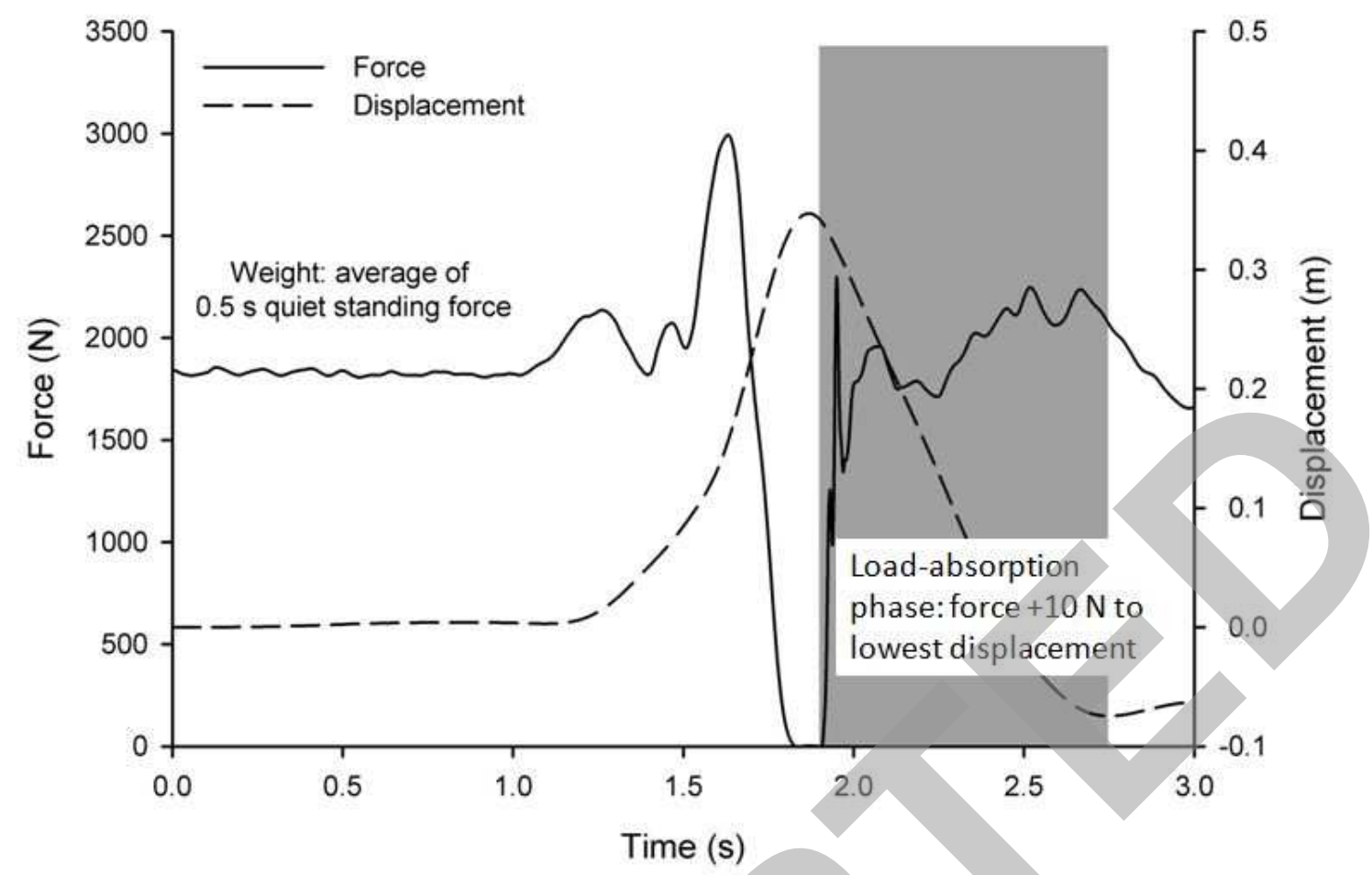




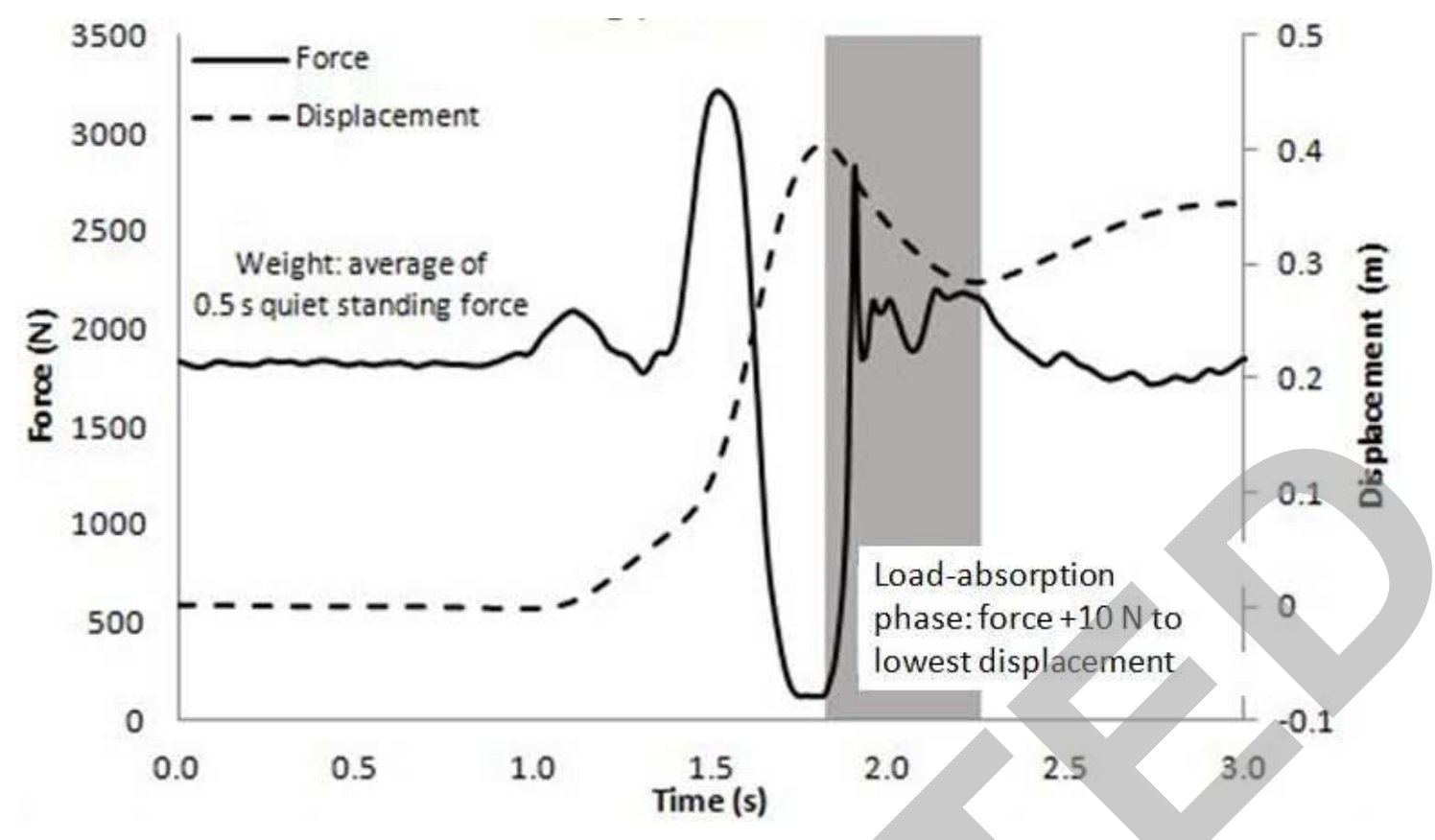




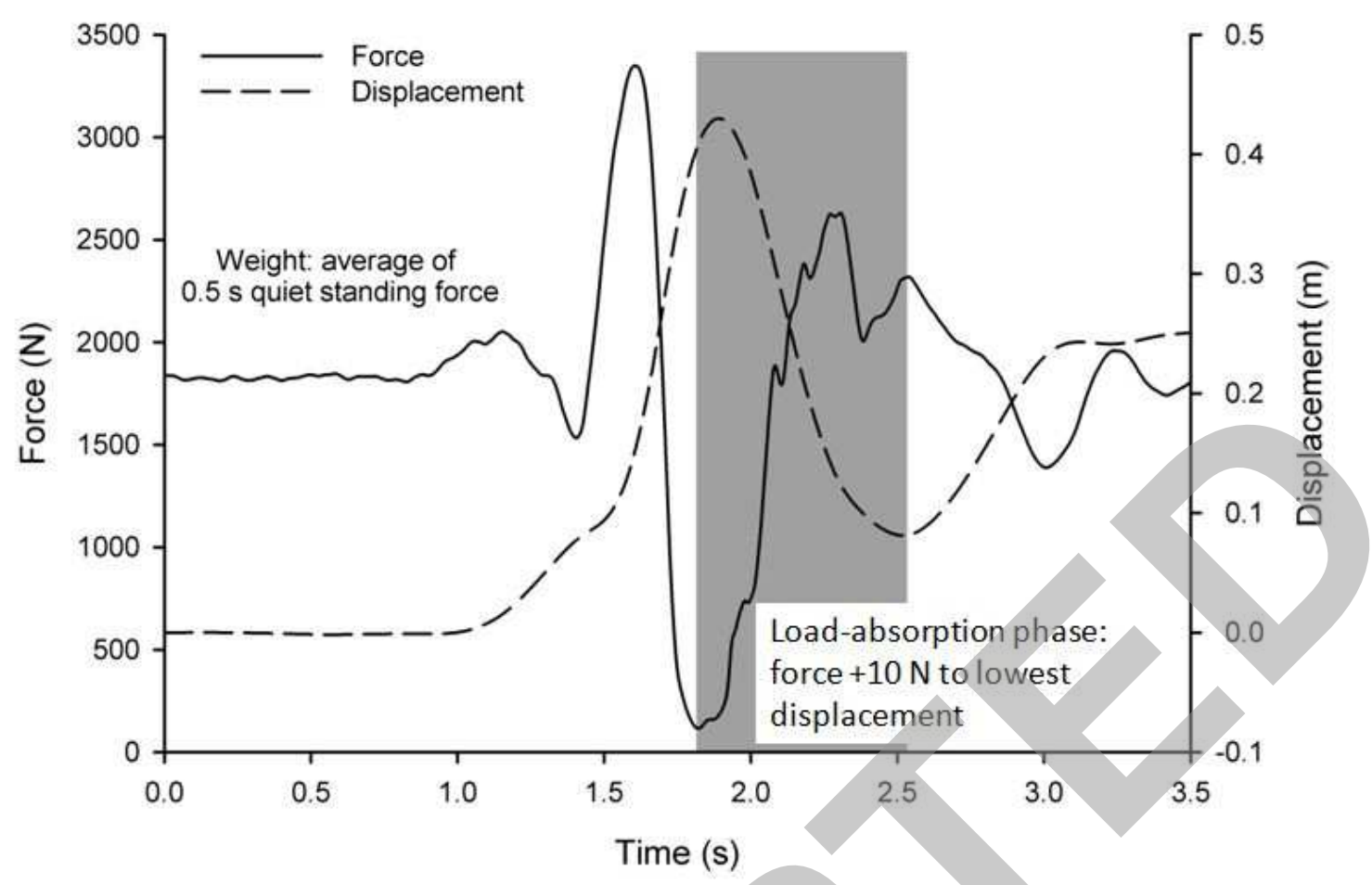




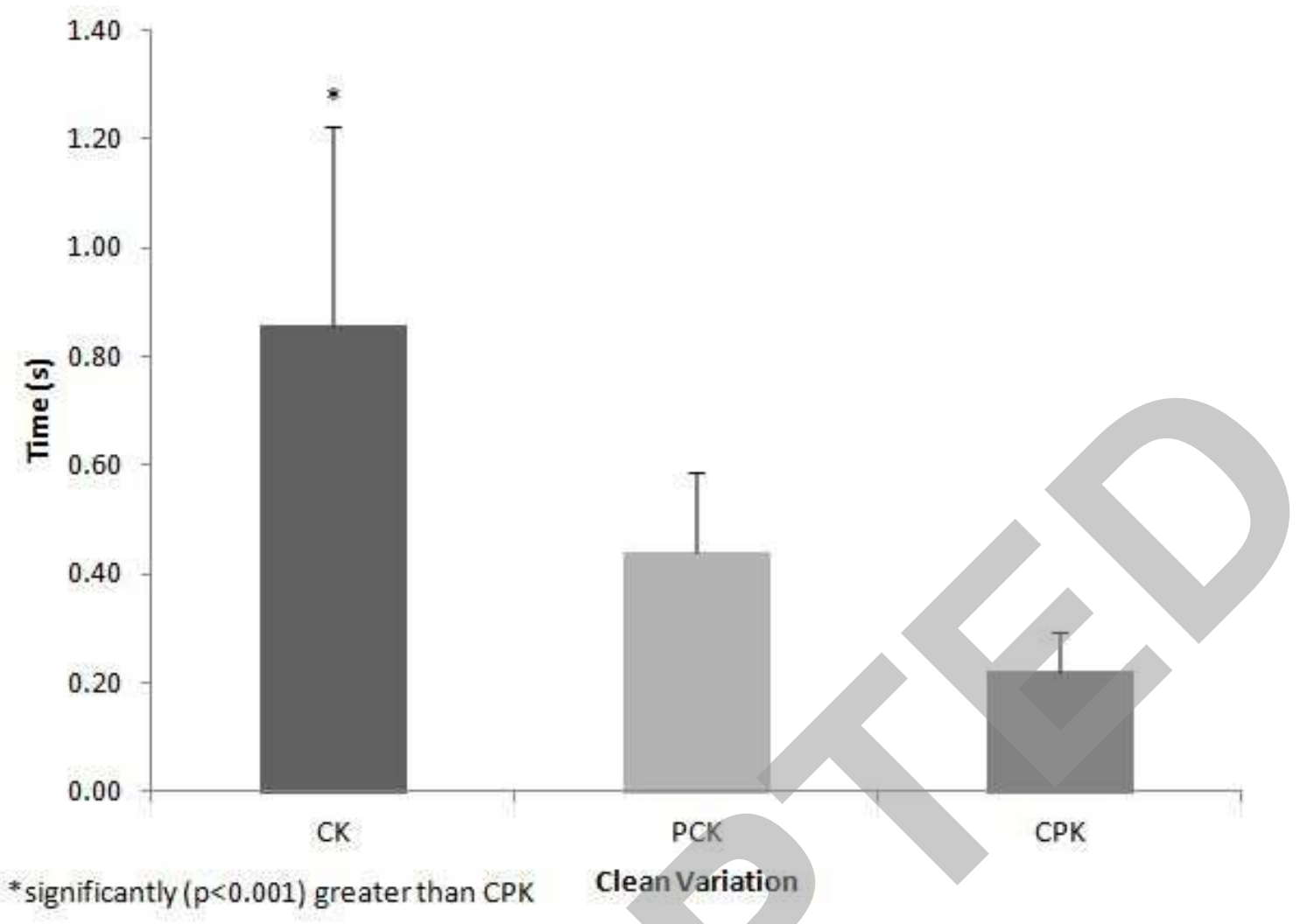




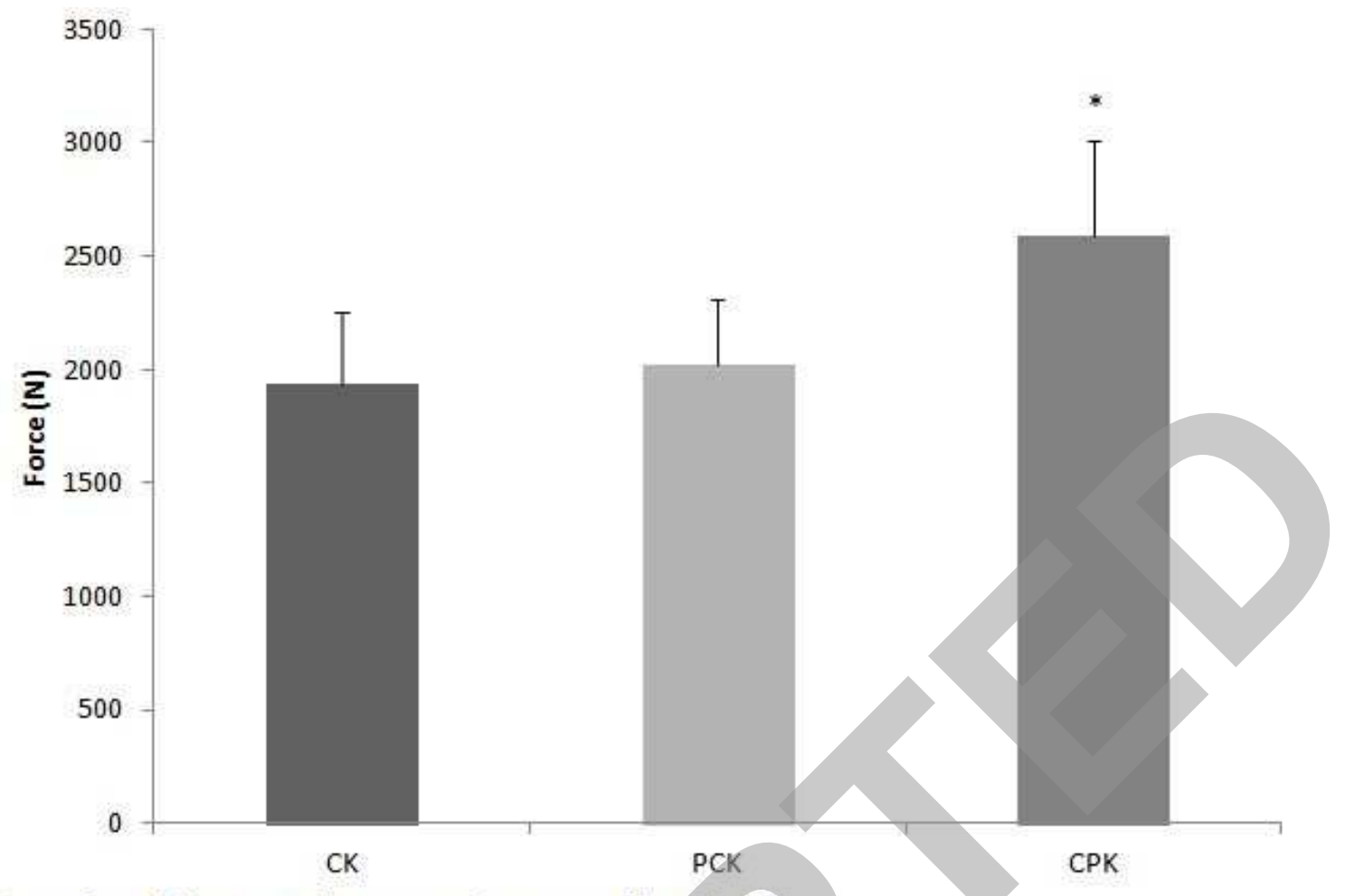

*significantly $(p=0.012)$ greater than PCK Clean Variation 


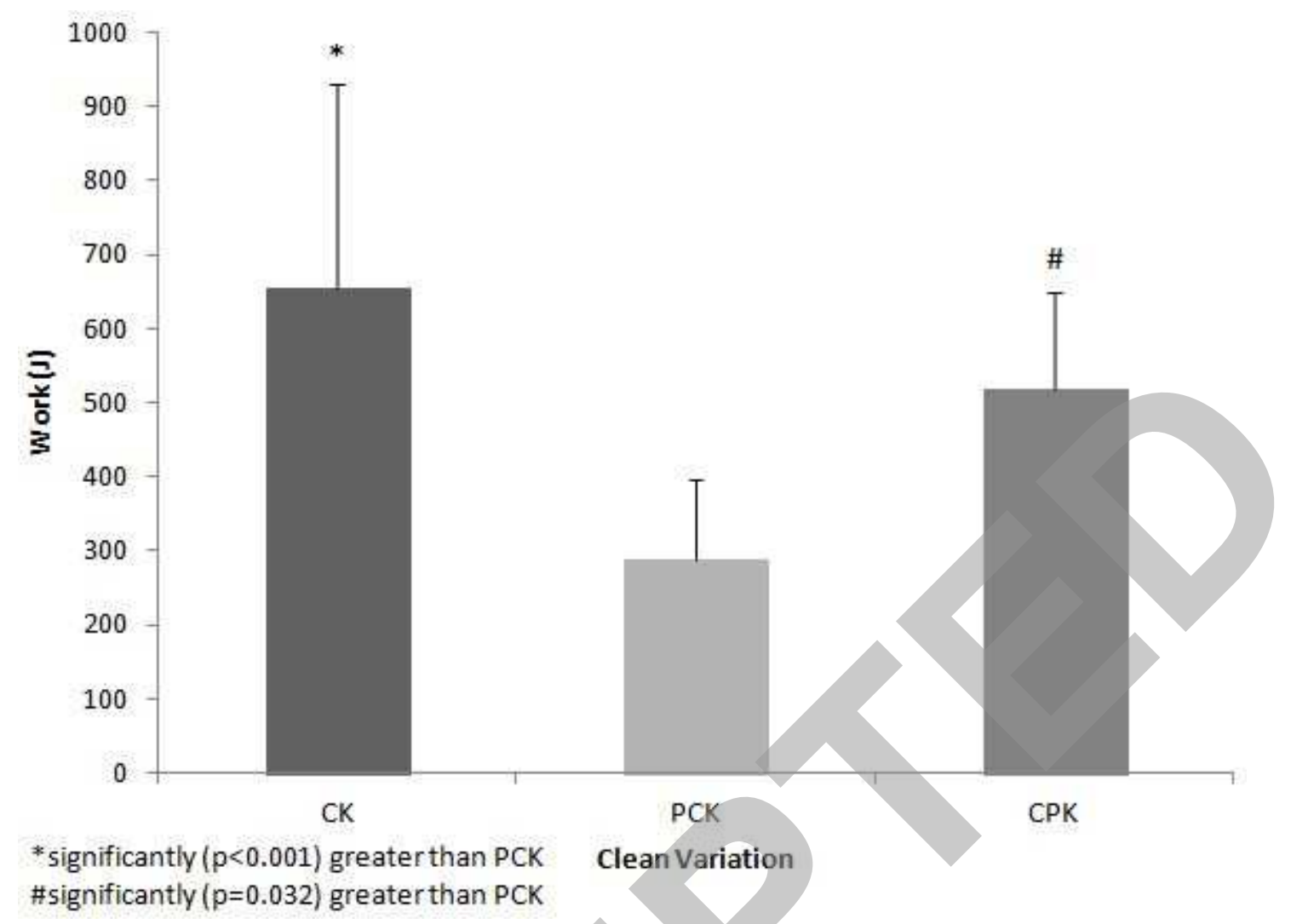

